\section{Front-Ends Are the Way to Go}

R.W. Poultney

People have been saying for the last five years that something exciting is happening in science publishing. The reality is somewhat different. While visionaries predicting the imminent downfall of the publishing establishment are almost too numerous to mention, it seems that little has been able to shake the status quo - yet. Does this mean that, despite the electronic communications revolution, nothing will change after all ? Nobody really knows, but it does seem a relatively safe bet that some kind of dramatic sea-change could still be just around the corner.

As many readers will know, ever since the beginning of the decade, new preprint initiatives (such as the famous Los Alamos preprint server http://xxx.lanl.gov/ pioneered by the string theorist Paul Ginsparg) have challenged the way particle physicists traditionally publish their results. As a matter of course, new papers are now routinely posted on the preprint servers for international distribution long before any kind of peer review or conventional publication has taken place.

International laboratories such as CERN have chosen to cease hard-copy mailing of preprints in favour of their own electronic systems (http://www.cern.ch/). There can also be little doubt that the new servers, along with electronic versions of some existing journals are here to stay (e.g., Elsevier Science's Nuclear Physics Electronic at http://www.elsevier.nl/ and, as from January 1996, 14 IOPP journals at http://www.iopp.org/, including Physics Express Letters.

But what do the changes really mean for the world of scientific publishing ? Paul Ginsparg believes that the technological developments will have some profound implications: "Since publication in a journal no longer gives a particularly useful guide, readers are forced to perform the majority of their selections by their own criteria. The current implementation of peer review should be entirely rethought in view of the new methods of

Roger Poultney, who trained as an experimental highenergy physicist, is a freelance science journalist and publishing editor based in Amsterdam. He was executive editor of Nuclear Physics B from 1991 to 1994, and is currently setting up new electronic journals for various European organizations. electronic publication and dissemination." While some scientists may argue that without quality control an unprecedented proliferation of information will ensue, others passionately believe that a fairer and more objective system of evaluation could emerge from the technological advances. There are even those who believe that freedom from any kind of censure can only be of benefit to the scientific community as a whole.

One proposal is to measure the amount of citations that preprints made available on databases receive, a philosophy which has been supported by several observers. The main drawback seems to be that papers which are controversial in nature (but not necessarily correct) invariably provoke an interested reaction, and initial citations alone are not necessarily a reliable indicator of quality. Neither, indeed, is traditional peer review, which is often seen as an arbitrary and subjective process. Ensuring a uniform standard is more-or-less impossible, and although some attempts have been made to measure the objectivity of reviewing procedures, there can be little doubt that any policy will have its shortcomings. Says Ginsparg: "One of the foremost problems at present is the large amount of information lost in the conventional peer review process, with the end result only a single, one-time, allor-nothing binary decision. Although this may somehow be adequate for the purpose of validating research for job and grant allocations, it clearly provides little benefit to the average reader."

\section{Second-tier Front-ends}

In reality, what will probably happen is the development some sort of "two-tier" system, where all works are systematically archived on the preprint databases (so that nothing is rejected erroneously), but with a form of indexed "front-end" guide to the literature which acts as a filter and takes readers directly to the most significant publications. The situation is neatly summed up by Ginsparg: "Since there are now no financial or physical barriers to widespread dissemination, we can imagine a relatively complete raw archive unfettered by any unnecessary delays in availability. Any type of information could be overlayed on this raw archive and maintained by any third parties."

This scenario has the natural advantage that while papers stored in the preprint archives are "written in stone", the front-end review systems are dynamic and can be changed to include papers retrospectively as fields and subject emphasis evolve. This is especially true in highenergy physics theory, where the impact of new ideas may only show up years or even decades after their construction. In this way the initial rejection of what are eventually realised to be classic papers (such as George Zwieg's 1963 work predicting the existence of quarks) should never happen. Good science will not be thrown away on the whims or prejudices of editors and referees, and the more democratic taste of the international community will be the force that prevails.

Several researchers and private organizations have sought to develop front-end systems along the new model advocated by Ginsparg. Examples include Paul Mende's Virtual Review (http://www.het. brown.edu/physics/review/index.html), run from Brown University in the USA, and Eagle Intermedia's new Particle Physics (http://193.78.164.43/av/edu/ppj/ home.html), which is based in Europe and run in close affiliation with Theory of Atomic and Molecular Clusters, a pilot electronic publication based at the University of Oldenburg. These systems work essentially on the same principle, with volunteer "referees" nominating those papers which they believe to be currently the most important in their respective fields. In the Virtual Review, papers are often cited as hypertext links appearing within the text of a general review of the field, whereas in Particle Physics, discrete lists of selected works are compiled on a bi-monthly basis (with hypertext links to signed reviews, where the referee does not wish to remain anonymous).

In any such front-end system, the issue of confidentiality is a difficult one, since while more open reviewing has its advocates, it does tend to discourage the criticism crucial to maintaining standards. It is for this reason that Particle Physics has chosen to experiment with both options, allowing referees the choice of anonymity or disclosure. Only through trial and error will the community discover which is to be preferred in this new world, since there are clearly pros and cons associated with both approaches. There is always the danger that partic- 
ipating individuals may end up visibly nominating papers for mainly political purposes. Worse still, it is not unknown for rival research groups to veto each others' results in order to gain the advantage of publishing first. But this is hardly a problem if both groups have already submitted their respective papers to the preprint archives; they are there for posterity (with their original submission dates), and it is the democratic opinion of the physics community, expressed through the front-end systems, which will ultimately decide on their relative impact.

\section{Some Reservations}

All physicists do not see this kind of approach as the way forward. David Fairlie, a theoretician at the University of Durham, remains unconvinced that frontend systems will have an impact. Says Fairlie: "The idea of nominations is a waste of time; indeed, when all physics articles [on the Web] carry abstracts there should be no need for a review at all! Increasingly, I believe people are finding and learning new ideas through conference participation, rather from the Internet, because they are a captive audience for the duration of a presentation."

Of course, there is little doubt that conventional peer review serves an important function in raising standards by improving papers through requested revisions. This process will still be required, though it seems possible that authors may arrange for it informally. It is certainly in their professional interests to have this kind of check before submitting to the preprint servers, where work becomes instantly accessible worldwide. Voluntary scrutiny already takes place with experimental papers produced by the large international collaborations. Here, an internal committee checks and double-checks the data, analysis, conclusions, and presentation before releasing the work. The result is that experimental papers rarely require any kind of revision before acceptance in the established journals. With more emphasis being placed on preprints as the "finally accepted form" of an author's work, theorists may well follow the trend and "self-police" their own papers. If this were to happen, standards could actually rise of their own accord. Far from leading to an uncontrolled explosion in mediocre preprints, the new developments might lead to an increase in quality, provided there is a widespreadendorsement of the new culture.

How this new culture takes off depends to a strong extent on existing tra- ditions within different scientific disciplines. Outsiders are very often surprised to learn that publishing traditions may vary greatly, even between closely related fields. Bertrand Duplantier, a theoretical physicist at CE Saclay in France collaborates with biologists on topics relating to DNA macromolecule statistics. As an active editor, Duplantier is intrigued by the differences in publishing culture between physicists and biologists. "Preprint distribution is already widely accepted in the high-energy physics community, and the new electronic systems have become very popular. But in biology there is a greater fear of plagiarism at the pre-publication stage, so preprint distribution is relatively less important."

From this it would seem that electronic publishing developments may take place at a different pace within even closely related scientific fields. But one thing that will promote new systems across the board will be rapid advances in communications technology. Ever more sophisticated World-Wide Web browsers are being released, and the ability to interpret and format data in a wide variety of input formats will help smooth over cultural differences. Besides handling HTML (the standard language for presenting material on the Web), browsers are now being developed that can process and display

\section{Soundings}

\section{No Growth for Physical Review}

In order to assure the long-term financial health of its major journals at a time when there is concern about declining subscriptions, the impact of electronic publications, growth in non-US submissions, and declining public and government support for science, the American Physical Society (APS) decided at its Council meeting last November to take measures to ensure that increases in library subscriptions to Physical Review remain at less than $10 \%$ p.a. for the next five years. Aside from the obvious cost reductions, there will be "no overall journal growth" by increasing acceptance standards at a time when the total number of submissions to say Phys Rev A-E and Letters increased by $9 \%$ between 1993 and 1994, and by $5 \%$ between 1994 and 1995 (submissions from eastern Europe increased by $63 \%$ and $52 \%$ in the same periods). Peter Adams, the Phys Rev B editor, writes in Notes to Authors that decisions on publications must now normally be reached after two referring rounds so that extended anonymous review cannot be used to transform an acceptable manuscript into a publishable one. The editors of Phys Rev E wish to publish papers that are not "merely new or free from error" but contain "substantial advancement of previously established knowledge". It is hoped that zero
$\mathrm{T}_{\mathrm{E}} \mathrm{X}$ and $\mathrm{LAT}_{\mathrm{E}} \mathrm{X}$ (the preferred typesetting language of mathematicians and particle physicists) as well as files from popular word-processing packages (which are often used to prepare less mathematical texts). These more flexible browsers will make the construction of on-line journals much simpler, with text conversion of articles to HTML becoming unnecessary. The age of the "do it yourself" journal really does seem to have arrived.

From all this, it can be seen that, whether the momentum for change is driven by technology, or by a real need for peer review reform, real opportunities to change things for the better clearly do exist. For this reason, it is important that the physicists give new systems their full and dedicated support: much will ultimately depend on the dedication and determination of individual practitioners. Only time will tell if those (Ginsparg included) who believe that the advantages make radical change inevitable are correct. However, one could well imagine that, without the active participation of senior figures in the new front-end systems, important new initiatives could wither and die. This would be a great pity for the physics community (which afterall invented WWW technology), and for science publishing in general.

overall growth can be achieved without a "page budget" for editors, but as higher standards increase submissions, this policy may have to be reviewed.

The APS has also decided to try to maintain a long-term policy for page charges that is the same for all fields following recent "experiments" that generated considerably instability. These mostly concerned the elimination of page charges for all submissions to Phys Rev D.The measure had the desired result of more submissions, but cost increases combined with the loss of page-charge income meant that $D$ ran at a loss, even following large increases to the subscription price; libraries which tend to buy packages of journals reacted negatively. The plan is now to raise page charges slightly on all non-compuscript submissions to all journals, and to have by 1999 zero page charges for compuscripts submitted to all Phys Rev journals (only Phys Rev $C$ and $D$ now have zero page charges for compuscripts).

Compuscripts will be redefined to include non$T_{E} X$ desktop wordprocessing formats as well files that are not used in the production process.

Historically, page charges were intended to cover editorial costs, and subscriptions the production and distribution costs, an approach (but not a policy) that was motivated by the desire not to become totally dependent on subscription income. The distinction between these activities has become rather fuzzy in an 